\title{
Formation of Planetary-Mass Brown Dwarfs in Magnetic Molecular Clouds
}

\author{
Alan P. Boss \\ Carnegie Institution of Washington, Department of Terrestrial \\ Magnetism, 5241 Broad Branch Road, N. W., Washington, D. C. \\ 20015-1305, U.S.A.
}

\begin{abstract}
Searches for very low mass objects in young star clusters have uncovered evidence for free-floating objects with inferred masses possibly as low as 5 to 15 Jupiter masses $\left(M_{J u p}\right)$, similar to the masses of several extrasolar planets. We show here that the process which forms single and multiple protostars, namely collapse and fragmentation of molecular clouds, might be able to produce self-gravitating objects with initial masses less than $\sim 1 M_{J u p}$. Models are calculated with a three dimensional, finite differences code which solves the equations of hydrodynamics, gravitation, and radiative transfer in the Eddington and diffusion approximations. Magnetic pressure is added to the gas pressure, magnetic tension is approximated by gravity dilution once collapse is well underway, and ambipolar diffusion is treated approximately as well. Initially oblate clouds fragment into multiple protostar systems containing a small number (of order four) of fragments. If such fragments can be ejected from an unstable quadruple protostar system, prior to gaining significantly more mass, protostellar collapse might then be able to explain the formation of free-floating objects with masses below $13 M_{J u p}$. These objects might then be best termed "sub-brown dwarfs".
\end{abstract}

\section{Introduction}

Brown dwarfs are readily detected in the field (Delfosse et al. 1999; Kirkpatrick et al. 2000; Leggett et al. 2000) and in young star clusters (Béjar, Zapatero Osorio, \& Rebolo 1999; Ardila, Martín, \& Basri 2000; Luhman et al. 2000; Najita, Tiede, \& Carr 2000). In the latter regions, isolated objects have been discovered (Lucas \& Roche 2000; Zapatero Osorio et al. 2000) with masses that may fall below the deuterium-burning limit of $\sim 13 M_{\text {Jup }}$. Radial velocity searches (e.g., Marcy, Butler, \& Vogt 2000; Udry et al. 2000) have detected about 100 very low mass companions to solar-type stars, with minimum masses in the range of $\sim 0.15 M_{J u p}$ to $\sim 15 M_{J u p}$. Apparently the least massive, isolated objects found in young star clusters may be less massive than the most massive planetary companions, possibly arguing against the use of a simple mass-based definition to distinguish between stars and planets. This raises the question of whether very low mass, free-floating objects can be formed directly by the star formation process, or whether they must form by a planetary formation process in orbit around a star, and later be ejected to interstellar space. 


\section{Limiting Mass Estimates}

Analytical Jeans mass estimates (Low \& Lynden-Bell 1976; Rees 1976; Silk 1977) of opacity-limited fragmentation suggested that the minimum protostellar mass might be in the range of $\sim 3 M_{J u p}$ to $\sim 7 M_{J u p}$. Numerical hydrodynamical calculations (Boss 1988, 1993) of protostellar collapse and fragmentation similarly produced a minimum protostellar fragment mass of $\sim 3 M_{\text {Jup }}$ to $\sim 10 M_{\text {Jup }}$. However, both of these types of estimates neglected magnetic fields, which would be expected to dampen fragmentation and increase the minimum protostellar mass, and also neglected subsequent accretion of gas from the infalling cloud envelope. These estimates thus seemed to place a robust lower bound on the minimum stellar mass of $\sim 10 M_{J u p}$.

Estimates of the maximum mass of a gas giant planet (Lin \& Papaloizou 1980) suggested that gap formation would prevent further accretion of gas once a gas giant planet reached a mass of $\sim 1 M_{J u p}$. This led to the expectation of a paucity of objects with masses in the range between $\sim 1 M_{J u p}$ and $\sim 10 M_{J u p}$, providing a convenient division between planets and brown dwarfs.

However, observations of planetary-mass companions to solar-type stars and of free-floating objects in young star clusters do not support the idea of a universal mass gap: objects with masses in the range of $\sim 1 M_{J u p}$ to $\sim 10 M_{J u p}$ are found in both locales. Calculations have shown that gap formation need not stop growth at a mass of $\sim 1 M_{J u p}$ in the core accretion scenario for gas giant planet formation (Bryden et al. 1999; Kley 2000). Furthermore, the alternative scenario of forming gas giant protoplanets rapidly through a disk instability (Boss 2000) seems to be able to form protoplanets directly with masses in the range of $\sim 1 M_{\text {Jup }}$ to $\sim 10 M_{\text {Jup }}$. The question remains whether objects in this mass range can also be formed by protostellar collapse.

\section{Numerical Models}

We present here a key model (Boss 2001) from a large suite of models of the collapse of magnetic dense cloud cores (Boss 2002). The models were calculated with a three-dimensional, radiative, self-gravitating hydrodynamics code with over one million grid points. The initial conditions were chosen to represent observed magnetically-supported, dense cloud cores (Ward-Thompson, Motte, $\&$ André 1999). The density profile is Gaussian in radius with a central density $\left(\rho_{o}=2 \times 10^{-18} \mathrm{~g} \mathrm{~cm}^{-3}\right) 20$ times higher than that at the boundary. The cloud is initially oblate with an axis ratio of $2: 1$, a radius of $R=1.0 \times 10^{17} \mathrm{~cm}$, and an initial temperature of $10 \mathrm{~K}$, yielding a mass of $2.1 \mathrm{M}_{\odot}$ and an initial ratio of thermal to gravitational energy of $\alpha_{i}=0.30$. Rotation at a rate of $10^{-14} \mathrm{rad} \mathrm{s}^{-1}$ produces an initial ratio of rotational to gravitational energy $\beta_{i}=1.1 \times 10^{-4}$.

The magnetic field pressure $B^{2} / 8 \pi$ is added to the gas pressure. The field strength $B$ depends on the gas density in dense cloud cores as $B=B_{o}\left(\rho / \rho_{o}\right)^{\kappa}$, where $\kappa \approx 1 / 2$ and $B_{o}$ is the field strength at a reference density $\rho_{o}$. The effects of ambipolar diffusion (e.g., Ciolek \& Mouschovias 1995) are approximated by specifying that $B_{o}=B_{o}(t)=B_{o i}\left(1-t / \tau_{A D}\right)$, where $B_{o i}$ is a constant equal to $200 \mu \mathrm{G}, t$ is the model time, and the ambipolar diffusion time scale $\tau_{A D}$ is 10 $t_{f f}$. The free fall time $t_{f f}=t_{f f}\left(\rho_{o}\right)=1.486 \times 10^{12} \mathrm{~s}$. With $B_{o i}=200 \mu \mathrm{G}$ at 
$n_{o}=6 \times 10^{5} \mathrm{~cm}^{-3}$, the model starts with a ratio of magnetic to gravitational energy of $\gamma_{i}=0.43$. Loss of magnetic field support by ambipolar diffusion leads to dynamic collapse after a few free fall times. Once collapse begins, and the central density exceeds $\sim 10^{-15} \mathrm{~g} \mathrm{~cm}^{-3}$, magnetic tension forces are included in an approximate manner by diluting the overall gravitational potential (e.g., Basu 1997).

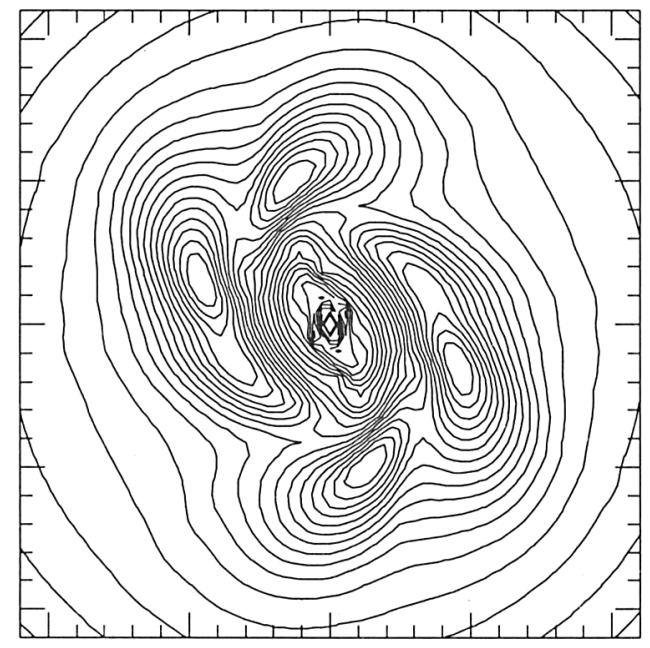

Fig. 1. Equatorial density for an initially oblate magnetic cloud core which has collapsed, rebounded, and fragmented into a quadruple protostar system with fragment masses less than a Jupiter-mass (Boss 2001). Decompressional cooling during the rebound lowers the Jeans mass to planetary masses. The region shown has a radius of $4.7 \times 10^{13} \mathrm{~cm}(3.1 \mathrm{AU})$. The maximum density of $6.3 \times 10^{-10} \mathrm{~g} \mathrm{~cm}^{-3}$ occurs in the off-center clumps. Contours represent changes by factors of 1.3. A density minimum occurs in the central regions of the cloud, where the gas is moving outward and cooling.

Figure 1 shows that the cloud collapses and fragments into a quadruple protostar system. Without magnetic tension forces, a central rebound and fragmentation do not occur for this cloud (Boss 2001). The fragments are gravitationally bound and have masses ranging from $\sim 0.3 M_{J u p}$ to $\sim 0.5 M_{J u p}$. These fragment masses are at least a factor of 10 times smaller than the lowest mass fragments obtained in non-magnetic collapses (Boss 1988, 1993). This occurs because magnetic tension forces lead to a central rebound and to decompressional cooling, and hence to lower temperatures. The Jeans mass depends on temperature to the $3 / 2$ power, so a decrease in temperature results in a significant reduction in the Jeans mass. 


\section{Conclusions}

The quadruple system shown in Figure 1 will disintegrate and eject one or two protostars that could become free-floating objects with a mass less than $13 M_{J u p}$, depending on the amount of subsequent gas accretion. Protostellar collapse might then explain the formation of planetary-mass, free-floating objects, obviating any need to invoke a more traditional planet formation process. Because these objects would form through the same process that forms dwarf stars and brown dwarfs, free-floating objects with masses in the range of 5 to $13 M_{J u p}$ could be denoted as "sub-brown dwarfs".

\section{References}

Ardila, D., Martín, E., \& Basri, G. 2000, AJ, 120, 479

Basu, S. 1997, ApJ, 485, 240

Béjar, V. J. S., Zapatero Osorio, M. R., \& Rebolo, R. 1999, ApJ, 521, 671

Boss, A. P. 1988, ApJ, 331, 370

- 1993, in The Realm of Interacting Binary Stars, ed. J. Sahade, G. McCluskey, Y. Kondo (Dordrecht: Kluwer), 355

- 2000, ApJ, 536, L101

—. 2001, ApJ, 551, L167

-. 2002, ApJ, 568, 743

Bryden, G., Chen, X., Lin, D. N. C., Nelson, R. P., \& Papaloizou, J. C. B. 1999, ApJ, 514, 344

Ciolek, G. E., \& Mouschovias, T. Ch. 1995, ApJ, 454, 194

Delfosse, X., et al. 1999, A\&AS, 135, 41

Kirkpatrick, J. D., et al. 2000, AJ, 120, 447

Kley, W. 2000, MNRAS, 313, L47

Leggett, S. K., et al. 2000, ApJ, 536, L35

Lin, D. N. C., \& Papaloizou, J. 1980, MNRAS, 191, 37

Low, C., \& Lynden-Bell, D. 1976, MNRAS, 176, 367

Lucas, P. W., \& Roche, P. F. 2000, MNRAS, 314, 858

Luhman, K. L., et al. 2000, ApJ, 540, 1016

Marcy, G. W., Butler, R. P., \& Vogt, S. S. 2000, ApJ, 536, L43

Najita, J. R., Tiede, G. P., \& Carr, J. S. 2000, ApJ, 541, 977

Rees, M. 1976, MNRAS, 176, 483

Silk, J. 1977, ApJ, 214, 152

Udry, S., et al. 2000, A\&A, 356, 590

Ward-Thompson, D., Motte, F., \& André, P. 1999, MNRAS, 305, 143

Zapatero Osorio, M. R., et al. 2000, Science, 290, 103 